\title{
The Role of Work in Breast Cancer Patients
}

\author{
Manuela Pennisi ${ }^{1}$, Giuseppe Chisari ${ }^{2}$, Marco Vacante ${ }^{3}$, Francesco Lucca $^{3}$, Salvatore Spitaleri ${ }^{3}$, \\ Giulia Malaguarnera ${ }^{4 *}$, Giuseppe Grosso ${ }^{4}$, Filippo Drago ${ }^{4}$, Vito Catania ${ }^{5}$, Alfredo Consoli ${ }^{5}$, \\ Mariano Malaguarnera ${ }^{3}$
}

\begin{abstract}
${ }^{1}$ Department of Neurosciences, University of Catania, Catania, Italy; ${ }^{2}$ Department of Biomedical Sciences, University of Catania, Catania, Italy; ${ }^{3}$ Research Center "The Great Senescence", University of Catania, Catania, Italy; ${ }^{4}$ International PhD Programme in Neuropharmacology, University of Catania, Catania, Italy; ${ }^{5}$ Department of General Surgery, University of Catania, Catania, Italy. Email: "giulia.malaguarnera@live.it
\end{abstract}

Received June $10^{\text {th }}, 2013$; revised July $9^{\text {th }}, 2013$; accepted July $17^{\text {th }}, 2013$

Copyright (C) 2013 Manuela Pennisi et al. This is an open access article distributed under the Creative Commons Attribution License, which permits unrestricted use, distribution, and reproduction in any medium, provided the original work is properly cited.

\begin{abstract}
Background: Since the survival rates of cancer have increased considerably, the long-term side effects of cancer and cancer-related treatments may impact survivors' capability to regain normal lives. The aim of this study was to evaluate the effects of the breast cancer on the job satisfaction and the quality of life. Methods: We enrolled 130 women divided into four groups: 1) 40 breast cancer survivors (aged 39 - 50); 2) 44 women diagnosed with breast cancer (aged 35 - 49); 3) 46 women in good health status (aged 37 - 48). Job satisfaction was measured with the Warr-Cook-Wall (WCW) Job satisfaction scale that measures overall job satisfaction and satisfaction with nine aspects of work. Results: Compared to healthy subjects, breast cancer survivors reported a poorer number of hours of work $(p<0.05)$ and a significant amount of variety in job ( $p<0.01)$, opportunity to use abilities $(\mathrm{p}<0.001)$, income $(\mathrm{p}<0.01)$. Compared to patients with breast cancer at diagnosis, breast cancer survivors reported a significant amount of variety in job (p $<$ $0.05)$, opportunity to use abilities ( $p<0.05)$, amount of responsibility $(p<0.05)$, income $(p<0.05)$. In our study, the comparison between breast cancer survivors, breast cancer at diagnosis, and healthy subjects does not differ significantly in overall job satisfaction. Conclusions: Understanding the job problems associated with cancer can provide relevant information regarding potential treatment and psychological support in breast cancer survivors.
\end{abstract}

Keywords: Breast Cancer; Job Satisfaction; Quality of Life; Cancer Survivors; Work; Absenteeism; Presenteeism

\section{Introduction}

Breast cancer is the most common malignancy in women with annual incidence rates ranging from 11.8 per 100,000 in eastern China to 86.3 per 100,000 in North America [1]. Early screening for breast cancer saves patient's breasts and improves their chances and full recovery [2]. Compared with women of similar age without a history of cancer, a slightly higher proportion of breast cancer survivors reduced work ability [3] or work performance. The prolonged work absenteeism has a relevant economic impact on the patient and her family. These outcomes can regrettably lead to a cascade of problems for the survivor, the workplace and the society [4]. Even in patients who have node-negative breast cancer and are considered to be at low risk, the 10 -year recurrence rate following exclusive local therapy is $15 \%$ $45 \%$, while in patients with node-positive disease, the

\footnotetext{
"Corresponding author.
}

rate reaches levels as high as $25 \%-90 \%$ [5]. Survivors may have ongoing emotional problems due to fears of recurrence or death and changes in social relationships [6]. Various studies demonstrated that exercise and physical activity can improve many of the symptoms and health problems experienced by breast cancer survivors $[7,8]$. The aim of this study was to evaluate the workdirected intervention on work productivity, on job satisfaction and consequently on absenteeism and presenteeism.

\section{Methods}

All eligible subjects to participate in this study were women, presenting for initial breast tumor evaluation at Cannizzaro Hospital from January 2008 to December 2010. Questionnaires were used to determine participants' demographic (age, education and marital status) and health information (non-cancer medical history, cancer disease stage at diagnosis, recurrence, cancer 
treatment and other medical information) (Table 1). The inclusion criteria were at least 30 years of age, employed full or part time. The exclusion criteria were as follows: patients who have alarming symptoms and signs, including weight loss, anaemia, headache; asthma or chronic obstructive pulmonary disease; chronic hepatitis or liver cirrhosis; angina, myocardial infarction or heart failure; malignancy; neurosis or psychosis; dementia. Participants were initially recruited to participate in a study examining work and ability and health related quality of life. After obtaining informed, written consent, demographic, laboratory and clinic-pathological data were obtained from medical record review and patient inter- view and incorporated into a clinical database. Job satisfaction was measured with the Warr-Cook-Wall (WCW) Job satisfaction scale developed by Warr [9]. The WCW instrument measures overall job satisfaction and satisfaction with nine aspects of work (amount of variety in job, opportunity to use abilities, freedom of working method, amount of responsibility, physical working condition, hours of work, income, recognition for work, and colleagues and fellow workers) with each item rated on a 7-point Likert scale $(1=$ extreme dissatisfaction to $7=$ extreme satisfaction). A higher overall mean score indicates higher job satisfaction. Absenteeism and presenteeism were evaluated in all subjects. Absenteeism was ex-

Table 1. Demographic characteristics of subjects included in the study.

\begin{tabular}{|c|c|c|c|}
\hline Characteristics & Breast Cancer Survivors (Group 1) & Breast Cancer at diagnosis (Group 2) & Healthy subjects (Group 3) \\
\hline Patients (n) & 40 & 44 & 46 \\
\hline Age (years) & $44.7 \pm 5.2$ & $41.8 \pm 6.7$ & $42.9 \pm 5.4$ \\
\hline HR (bpm) & $74 \pm 5$ & $77 \pm 6$ & $76 \pm 4$ \\
\hline $\mathrm{SBP}(\mathrm{mmHg})$ & $132 \pm 12$ & $136 \pm 8$ & $135 \pm 6$ \\
\hline $\mathrm{DBP}(\mathrm{mmHg})$ & $80 \pm 8$ & $78 \pm 9$ & $79 \pm 8$ \\
\hline $\operatorname{BMI}\left(\mathrm{Kg} / \mathrm{m}^{2}\right)$ & $25.8 \pm 2.4$ & $26.2 \pm 2.0$ & $25.7 \pm 2.6$ \\
\hline \multicolumn{4}{|l|}{ Education } \\
\hline$<$ High School & $12(30 \%)$ & $14(31 \%)$ & $12(26 \%)$ \\
\hline High School & $18(45 \%)$ & $18(40 \%)$ & $14(30 \%)$ \\
\hline$>$ High School & $10(25 \%)$ & $12(27 \%)$ & $20(43 \%)$ \\
\hline \multicolumn{4}{|l|}{ Marital status } \\
\hline Married & $24(60 \%)$ & $27(61 \%)$ & $28(60 \%)$ \\
\hline Single & $6(15 \%)$ & $4(9 \%)$ & $6(13 \%)$ \\
\hline Divorced/Separated & $4(10 \%)$ & $6(14 \%)$ & $4(8 \%)$ \\
\hline Widow/Widower & $6(15 \%)$ & $7(15 \%)$ & $8(17 \%)$ \\
\hline \multicolumn{4}{|l|}{ Employment } \\
\hline Working full time & $30(75 \%)$ & $31(70 \%)$ & $30(65 \%)$ \\
\hline Working part time & $10(25 \%)$ & $13(29 \%)$ & $16(35 \%)$ \\
\hline \multicolumn{4}{|l|}{ Smoking } \\
\hline No & $15(37 \%)$ & $12(27 \%)$ & $12(26 \%)$ \\
\hline Current & $20(50 \%)$ & $22(50 \%)$ & $18(39 \%)$ \\
\hline Ex & $5(12 \%)$ & $10(23 \%)$ & $16(35 \%)$ \\
\hline \multicolumn{4}{|l|}{ Alcohol } \\
\hline No & $22(55 \%)$ & $25(56 \%)$ & $26(56 \%)$ \\
\hline Current & $4(10 \%)$ & $6(14 \%)$ & $4(8 \%)$ \\
\hline Ex & $14(35 \%)$ & $13(29 \%)$ & $16(35 \%)$ \\
\hline
\end{tabular}


pressed as both the total work time lost (the sum of total hours absent for breast cancer symptoms) and the percentage of work time lost during employed time for the past seven days. Presenteeism was expressed as both total limited work time and the percentage of limited productivity whilst at work [10].

\section{Statistical Analysis}

Results are expressed as means $+/-$ standard deviations. Comparisons of quantitative data were made using the Student's t-test or Mann-Whitney test. Qualitative data were analyzed using the chi-square test. A P value of $<0.05$ was considered as indicating a statistically significant difference. All data management and statistical calculations were performed using SPSS 15.0 statistical package (Chicago, IL).

\section{Results}

Demographic characteristics of subjects included in the study are reported in Table 1 . There were not significant differences between groups at baseline with respect to sex, age, education, marital status, type of employment (full or part time), smoking and alcohol consumption. Subjects working full time were $75 \%$ in Group 1, 70\% in Group 2 and 65\% in Group 3; subjects working part time were in the three groups respectively $25 \%, 29 \%$ and $35 \%$.

Compared to healthy subjects, breast cancer survivors reported a poorer numbers of hours of work $(p<0.05)$ and a significant amount of variety in job ( $p<0.01)$, opportunity to use abilities $(\mathrm{p}<0.001)$, income $(\mathrm{p}<0.01)$. Compared to patients with breast cancer at diagnosis, breast cancer survivors reported a significant amount of variety in job $(\mathrm{p}<0.05)$, opportunity to use abilities ( $\mathrm{p}<$ $0.05)$, amount of responsibility $(\mathrm{p}<0.05)$, income $(\mathrm{p}<$ 0.05) (Table 2). Both the mean absenteeism and mean presenteeism were similar in all groups. In breast cancer survivors the overall job satisfaction was significantly increased in married and employed working full time. In patients with breast cancer at diagnosis the overall job satisfaction was significantly decreased in both married and working part-time subjects.

\section{Discussion}

The improvement of social environmental condition, medical care, screening and diagnostic parameters caused an increase of survival in breast cancer patients [11]. Since the survival rates of cancer have increased considerably, the long-term side effects of cancer and cancerrelated treatments may impact survivors' capability to regain normal lives. This implies that some forms of cancer are becoming chronic diseases entailing both poorer overall quality of life compared to the general population and disabling long-term residual symptoms, such as fatigue, depression, pain and functional limitation [12]. This condition determines a high impact on the quality of life, including, physical and mental health, on work and return to work. A large body of psychosocial cancer research has noted the importance of distressing factors, individual coping attempts, psychosocial burden and resources in understanding both health-related quality of life and psychological adjustment to cancer. The objective of our study was to evaluate the elements that have the main impact on overall job satisfaction separated from breast cancer survivors, breast cancer, and healthy workers. Advances in diagnosis and treatment of breast cancer have created a large population of survivors

Table 2. Job satisfaction scores in breast cancer survivors.

\begin{tabular}{|c|c|c|c|}
\hline & $\begin{array}{l}\text { Breast Cancer Survivors } \\
\quad(\text { Group } 1 ; \mathrm{n}=40)\end{array}$ & $\begin{array}{l}\text { Breast Cancer at diagnosis } \\
\quad(\text { Group } 2 ; n=44)\end{array}$ & Healthy subjects (Group 3; $n=46$ ) \\
\hline Amount of variety in job & $5.45 \pm 1.21$ & $4.87 \pm 1.44^{* *}$ & $4.76 \pm 1.61^{\mathrm{B}}$ \\
\hline Opportunity to use abilities & $5.87 \pm 1.04$ & $5.21 \pm 1.08^{* *}$ & $5.04 \pm 1.02^{\mathrm{C}}$ \\
\hline Freedom of working method & $5.21 \pm 1.44$ & $5.44 \pm 1.09^{*}$ & $5.10 \pm 1.10^{\mathrm{A}}$ \\
\hline Amount of responsibility & $5.75 \pm 1.67$ & $5.10 \pm 1.22^{* *}$ & $5.21 \pm 1.36^{\mathrm{A}}$ \\
\hline Physical working condition & $4.82 \pm 1.87$ & $5.00 \pm 1.46^{*}$ & $5.12 \pm 1.22^{\mathrm{A}}$ \\
\hline Hours of work & $4.67 \pm 1.44$ & $4.96 \pm 1.38^{*}$ & $5.28 \pm 1.20^{\mathrm{B}}$ \\
\hline Income & $4.44 \pm 1.65$ & $5.24 \pm 1.31^{* *}$ & $5.31 \pm 1.41^{\mathrm{B}}$ \\
\hline Recognition for work & $5.04 \pm 1.20$ & $5.10 \pm 1.28^{*}$ & $5.25 \pm 1.32^{\mathrm{A}}$ \\
\hline Colleagues and fellow workers & $5.61 \pm 1.08$ & $5.44 \pm 1.12^{*}$ & $5.36 \pm 1.18^{\mathrm{A}}$ \\
\hline Overall job satisfaction & $5.41 \pm 1.04$ & $5.20 \pm 1.18^{*}$ & $5.28 \pm 1.25^{\mathrm{A}}$ \\
\hline
\end{tabular}

Comparison between group 1 and group $2:{ }^{*} \mathrm{p}=\mathrm{NS} ;{ }^{* *} \mathrm{p}<0.05 ;{ }^{* * *} \mathrm{p}<0.001$. Comparison between group 1 and group $3:{ }^{\mathrm{A}} \mathrm{p}=\mathrm{NS} ;{ }^{\mathrm{B}} \mathrm{p}<0.05 ;{ }^{\mathrm{C}} \mathrm{p}<0.001$. 
requiring long-term follow-up care [1,2]. The risk of disease recurrence peaks during the first 5 years after primary therapy, but may continue for up to 30 years after that. Work plays an important role in the psychological treatment for many patients. As a result, breast cancer is increasingly viewed as a chronic illness [9-11]. Our culture continues to perpetuate the healthy subject view that an individual with cancer is somehow now defective. Work intervention has been found to have significant positive effects on functional capacity and physical functioning, fatigue, body image, psychological adjustment, sleep problems and overall health and quality of life [1216]. In our study, the comparison between breast cancer survivors, breast cancer at diagnosis, and healthy subjects does not differ significantly in overall job satisfaction. Job satisfaction was often stated as a strong motivator for social status and for social contacts. Changes in the life of the person with cancer (e.g. being away from work or not being able to do the usual roles at home) may affect the person's self-esteem [2]. Yet employers continue to perceive cancer survivors as poor risks for advancement and cancer survivors are at high risk for job loss. A limitation of this study was the small number of included subjects. Understanding job problems associated with cancer can provide relevant information regarding potential treatment and psychological support in breast cancer survivors. More systematic investigation is needed varying the dimensions of activity explored in this study.

\section{Acknowledgements}

GM was supported by the International $\mathrm{PhD}$ programme in Neuropharmacology, University of Catania. The authors declare that they have no competing interests.

\section{REFERENCES}

[1] D. M. Parkin, F. Bray, J. Ferlay and P. Pisani, "Global Cancer Statistics, 2002," CA: A Cancer Journal for Clinicians, Vol. 55, No. 2, 2005, pp. 74-108. http://dx.doi.org/10.3322/canjclin.55.2.74

[2] L. L. Humphrey, M. Helfand, B. K. Chan and S. H. Woolf, "Breast Cancer Screening: A Summary of the Evidence for the US Preventive Services Task Force," Annals of Internal Medicine, Vol. 137, No. 5, 2002, pp. 347360.

http://dx.doi.org/10.7326/0003-4819-137-5 Part 1-20020 9030-00012

[3] C. J. Bradley, D. Neumark, H. L. Bednarek and M. Schenk, "Short-Term Effects of Breast Cancer on Labor Market Attachment: Results from a Longitudinal Study," Journal of Health Economics, Vol. 24, No. 1, 2005, pp. 137-160. http://dx.doi.org/10.1016/j.jhealeco.2004.07.003

[4] C. Messner and D. Patterson, "The Challenge of Cancer in the Workplace," Cancer Practice, Vol. 9, No. 1, 2001, pp. $50-51$. http://dx.doi.org/10.1046/j.1523-5394.2001.91009.x

[5] I. A. Olivotto, C. D. Bajdik, P. M. Ravdin, C. H. Speers, A. J. Coldman, B. D. Norris, G. J. Davis, S. K. Chia and K. A. Gelmon, "Population-Based Validation of the Prognostic Model ADJUVANT! for Early Breast Cancer," Journal of Clinical Oncology, Vol. 23, No. 12, 2005, pp. 2716-2725. http://dx.doi.org/10.1200/JCO.2005.06.178

[6] N. K. Aaronson, "Methodologic Issues in Assessing the Quality of Life of Cancer Patients," Cancer, Vol. 67, Suppl. 3, 1991, pp. 844-850.

http://dx.doi.org/10.1002/1097-0142(19910201)67:3+<84 4::AID-CNCR2820671416>3.0.CO;2-B

[7] V. Mock, K. H. Dow, C. J. Meares, P. M. Grimm, J. A. Dienemann, M. E. Haisfield-Wolfe, W. Quitasol, S. Mitchell, A. Chakravarthy and I. Gage, "Effects of Exercise on Fatigue, Physical Functioning, and Emotional Distress during Radiation Therapy for Breast Cancer," Oncology Nursing Forum, Vol. 24, No. 6, 1997, pp. 991-1000.

[8] A. J. Daley, H. Crank, N. Mutrie, J. M. Saxton and R. Coleman, "Determinants of Adherence to Exercise in Women Treated for Breast Cancer," European Journal of Oncology Nursing, Vol. 11, No. 5, 2007, pp. 392-399. http://dx.doi.org/10.1016/j.ejon.2007.03.001

[9] P. Warr, J. Cook and T. Wall, "Scales for the Measurement of Some Work Attitudes and Aspects of Psychological Well-Being," Journal of Occupational Psychology, Vol. 52, No. 2, 1979, pp. 129-148. http://dx.doi.org/10.1111/j.2044-8325.1979.tb00448.x

[10] M. C. Reilly, K. L. Gooch, R. L. Wong, H. Kupper and D. van der Heijde, "Validity, Reliability and Responsiveness of the Work Productivity and Activity Impairment Questionnaire in Ankylosing Spondylitis," Rheumatology (Oxford), Vol. 49, No. 4, 2010, pp. 812-819.

[11] M. Vacante, V. D’Agata, M. Motta, G. Malaguarnera, A. Biondi, F. Basile, M. Malaguarnera, C. Gagliano, F. Drago and S. Salamone, "Centenarians and Supercentenarians: A Black Swan. Emerging Social, Medical and Surgical Problems," BMC Surgery, Vol. 12, Suppl. 1, 2012, p. S36. http://dx.doi.org/10.1186/1471-2482-12-S1-S36

[12] P. Frazzetto, M. Vacante, M. Malaguarnera, E. Vinci, F. Catalano, E. Cataudella, F. Drago, G. Malaguarnera, F. Basile and A. Biondi, "Depression in Older Breast Cancer Survivors," BMC Surgery, Vol. 12, Suppl. 1, 2012, p. S14. http://dx.doi.org/10.1186/1471-2482-12-S1-S14

[13] V. S. Helgeson, "Recent Advances in Psychosocial Oncology," Journal of Consulting and Clinical Psychology, Vol. 73, No. 2, 2005, pp. 268-271. http://dx.doi.org/10.1037/0022-006X.73.2.268

[14] V. S. Helgeson and P. L. Tomich, "Surviving Cancer: A Comparison of 5-Year Disease-Free Breast Cancer Survivors with Healthy Women," Psycho-Oncology, Vol. 14, No. 4, 2005, pp. 307-317. http://dx.doi.org/10.1002/pon.848

[15] S. Moulder and G. N. Hortobagyi, "Advances in the Treatment of Breast Cancer," Clinical Pharmacology \& Therapeutics, Vol. 83, 2008, pp. 26-36. http://dx.doi.org/10.1038/sj.clpt.6100449

[16] F. C. Dimeo, F. Thomas, C. Raabe-Menssen, F. Pröpper and M. Mathias, "Effect of Aerobic Exercise and Relaxa- 
tion Training on Fatigue and Physical Performance of Cancer Patients after Surgery. A Randomised Controlled Trial," Supportive Care in Cancer, Vol. 12, No. 11, 2004, pp. 774-779.

http://dx.doi.org/10.1007/s00520-004-0676-4 